Ann. Biol. anim. Bioch. Biophys., r972, 12 (2), 307-320.

\title{
INFLUENCE DE LA TENEUR EN URÉE DU RÉGIME SUR L'AMINOACIDÉMIE LIBRE DE CHEVRES EN LACTATION
}

\author{
C. CHAMPREDON et R. PION \\ avec la collaboration technique de Marie-Claude Valluy, A. SElle, \\ G. Bayle et J. Prugnaud \\ Station d'Études des Métabolismes, \\ Centre de Recherches de Clermont-Ferrand, I. N. R. A., \\ 63 - Saint-Genès-Champanelle
}

\section{RÉSUMÉ}

Huit chèvres en lactation ont reçu pendant des périodes de 5 à 6 semaines des régimes comportant du foin et des concentrés isoénergétiques à base d'orge, contenant de l'urée, ou du tourteau d'arachide, ou un mélange des deux. Les concentrés distribués au cours des périodes successives contenaient respectivement I, 2, 3 et o p. Ioo d'urée; le concentré à $3 \mathrm{p}$. Ioo d'urée ne contenait pas de tourteau d'arachide, alors que les autres en renfermaient une quantité telle que les régimes soient isoazotés, le foin représentait le tiers de la ration totale. A la fin de chaque période expérimentale, les consommations d'aliments et les productions laitières étaient mesurées, et des prélèvements de sang étaient effectués en vue de la détermination de la glycémie, de l'urémie et des teneurs du sang en acides aminés libres, dosés par chromatographie sur colonne échangeuse d'ions.

Les consommations d'aliments ont été très variables au cours des périodes expérimentales successives; le concentré contenant $2 \mathrm{p}$. Ioo d'urée a été bien accepté. Les productions laitières n'étaient pas très élevées, alors que les gains de poids des animaux étaient relativement importants, ce qui peut résulter de la proportion élevée de céréale dans les rations ingérées par les chèvres. Les urémies et les glycémies étaient minimales à la fin de la période correspondant à la distribution du concentré contenant 2 p. Ioo d'urée. Les variations de concentrations des acides aminés libres du sang semblent davantage résulter du taux de satisfaction des besoins azotés et énergétiques des animaux, que de la nature du complément azoté, à quelques exceptions près : les teneurs en glutamine du sang des animaux recevant de l'urée étaient élevées, ce qui peut être dû à une augmentation du passage d'ammoniac du rumen dans le sang. Le mélange orge-arachideurée contenant 2 p. Ioo d'urée a paru être le mieux adapté aux besoins des animaux dans le cas de ce type de régime particulièrement favorable à l'utilisation de formes solubles de l'azote.

\section{INTRODUC'TION}

L'urée est de plus en plus utilisée dans l'alimentation des ruminants et de nombreuses mises au point telles que celles de Briggs (I967), Armstrong et Trinder (I966), Chalupa (I968), Olitjen (I969), Helmer et Bartley (I97I) passent en 
revue les divers travaux concernant son utilisation. En effet, les ruminants peuvent satisfaire au moins une partie notable de leurs besoins à partir de formes simples d'azote, grâce aux synthèses d'acides aminés, et, en particulier, d'acides aminés indispensables, qui peuvent être effectuées par les bactéries du rumen pour la constitution de leurs protéines. Cette synthèse peut être réalisée notamment à partir de l'ammoniac qui provient de la dégradation de l'urée ; elle est favorisée par la présence d'une source convenable d'énergie, en particulier sous forme de céréales.

La satisfaction des besoins en acides aminés " indispensables " des ruminants dépend des quantités de chacun d'eux qui sont mises à la disposition de leurs tissus à la suite des processus de la digestion, en particulier dans le rumen. Ellle peut être estimée, d'une manière indirecte, grâce à l'étude des teneurs en acides aminés libres du sang. En effet, les acides aminés provenant de la digestion sont absorbés au moins en majeure partie sous forme libre à partir du tractus gastro-intestinal et passent dans le sang. Ils sont transportés par la veine porte vers le foie où certains d'entre eux peuvent subir des transformations et, en particulier, être utilisés pour la néoglucogénèse (EGAN et BLACK, I968; EGAN, MOLLER et BLACK, I970). Les autres atteignent le sang périphérique où ils circulent sous forme libre pour être transportés vers les lieux d'utilisation, dans la glande mammaire par exemple (VERBECKE et PEETERS, I965; MEPHAM et LINZELL, I966). Les concentrations des acides aminés libres du sang dépendent, à chaque instant, de la différence entre les apports, exogènes et endogènes, et les diverses utilisations, et peuvent donc constituer un test de l'état de satisfaction des besoins des animaux. C'est pourquoi nous avons étudié, pour déterminer l'effet de la substitution de l'urée à du tourteau d'arachide sur la nutrition azotée des chèvres laitières, l'influence du régime sur les concentrations en acides aminés libres du sang.

\section{MATÉRIEL ET MÉTHODES}

\section{Animaux et régimes alimentaires}

Nous avons utilisé huit chèvres de race Alpine Chamoisée. Les animaux ont été répartis en deux lots de la manière suivante :

- lot A : Les quatre chèvres ayant produit la plus grande quantité de lait durant les 28 semaines de l'expérience ( $447 \mathrm{~kg}$ en moyenne);

- lot B : Les quatre autres chèvres (3II $\mathrm{kg}$ en moyenne).

Nous avons distribué aux animaux les aliments suivants :

- du foin de graminées de moyenne qualité, haché assez long, distribué matin et soir et en quantité correspondant au tiers de la ration totale ;

- de l'orge sous forme de granulés distribués au milieu de la journée à raison de $650 \mathrm{~g}$ par animal durant toute la durée de l'expérience ;

- les concentrés expérimentaux (granulés) distribués après la traite du matin et avant celle du soir, dont les compositions (tabl. I) ont été établies de manière que les quatre formules soient sensiblement isoénergétiques et isoazotées.

Les quantités de foin et de concentrés expérimentaux étaient déterminées en fonction de la production laitière de la semaine précédente et distribuées en léger excès par rapport aux besoins d'entretien et de production des animaux. Le rationnement a été établi selon les données de FeHr et Disset (I969). 


\section{TABLEAU I}

Composition des concentrés expérimentaux

(en $\mathrm{g} / \mathrm{kg}$ de matière brute)

\begin{tabular}{|c|c|c|c|c|}
\hline Aliment & $\mathrm{U}-\mathbf{0}$ & $\mathrm{U}-\mathbf{1}$ & $\mathrm{U}-2$ & $\mathrm{U}-3$ \\
\hline Urée & 0 & 10 & 20 & 30 \\
\hline Tourteau d'arachide $\ldots \ldots \ldots \ldots$ & 150 & 90 & 40 & 0 \\
\hline Mélange minéral vitaminisé .... & 50 & 50 & 50 & 50 \\
\hline Mélasse de canne $\ldots \ldots \ldots \ldots \ldots$ & 50 & 50 & 50 & 50 \\
\hline Farine de Juzerne déshydratée. . & 100 & 100 & 100 & 100 \\
\hline Orge $\ldots \ldots \ldots \ldots \ldots \ldots \ldots$ & 650 & 700 & 740 & 770 \\
\hline $\mathrm{UF} / \mathrm{kg}$ & 0,90 & 0,89 & 0,88 & 0,87 \\
\hline Azote total $(g) \ldots \ldots \ldots \ldots \ldots$ & 27,4 & 27,4 & 28,0 & 29,3 \\
\hline Azote uréique $\%$ de l'azote total & 0 & 15,3 & 30,0 & 42,9 \\
\hline
\end{tabular}

\section{Déroulement de l'expérience}

L'expérience qui comportait cinq périodes, a débuté lorsque toutes les chèvres étaient en lactation depuis au moins deux semaines et au plus dix semaines. Les animaux ont reçu successivement les quatre concentrés expérimentaux (tabl. 2). La succession des 3 premières périodes a été choisie de manière à adapter progressivement les animaux à recevoir des proportions croissantes d'urée dans le concentré. Le début de chacune des quatre premières périodes a servi de transition en vue d'adapter les animaux au nouvel aliment.

\section{TABLEAU 2}

\section{Schéma expérimental}

\begin{tabular}{c|c|c|c|c|c}
\hline \hline Période expérimentale & 1 & 2 & 3 & 4 & 5 \\
\hline Concentré distribué & $\mathrm{U}-1$ & $\mathrm{U}-2$ & $\mathrm{U}-3$ & $\mathrm{U}-0$ & $\mathrm{U}-0$ \\
\hline Durée de la période (jours) & 42 & 35 & 39 & 38 & 42 \\
\hline
\end{tabular}

\section{Mesures et analyșes effectuées}

\section{Aliments ingérés}

Dans toute l'expérience, les aliments ont été pesés avant leur distribution, mais ce n'est que durant les deux dernières semaines de chaque période, c'est-à-dire lorsque les animaux étaient bien adaptés aux concentrés expérimentaux étudiés, que l'on a pesé les refus en vue de la détermination des quantités d'aliments ingérées. Les moyennes de consommation ont été établies à partir des mesures effectuées pendant les 5 premiers jours de la dernière semaine de chaque période. 


\section{Production laitière}

Le lait produit par chaque chèvre a été pesé à chaque traite. Durant la dernière semaine de chaque période, un échantillon représentatif du lait produit par chaque animal au cours de dix traites consécutives a été conservé en vue de la détermination du taux butyreux par la méthode Gerber et du taux des matières azotées par la méthode au noir amido. .

\section{Poids des animaux}

Les pesées ont été effectuées chaque semaine en milieu d'après-midi.

\section{Prélèvements de sang}

Le sang nécessaire à la détermination des concentrations sanguines en urée, glucose et acides aminés libres, a été prélevé à la seringue dans la veine jugulaire de chaque animal. Les prélèvements ont été effectués durant la dernière semaine de chaque période expérimentale, par deux fois le même jour, en milieu de matinée et d'après-midi. Le sang était recueilli dans 7 fois son volume d'éthanol $95^{\circ}$ froid et conservé à $-15^{\circ} \mathrm{C}$. Les échantillons ont été regroupés par période expérimentale et par lot d'animaux dès que les productions laitières mesurées sur les 28 semaines de l'expérience ont pu être déterminées.

\section{Méthodes analytiques}

Les acides aminés, le glucose et l'urée étaient extraits avec l'éthanol $82^{\circ}$ froid par épuisements successifs. Une fraction de l'extrait éthanolique était utilisée pour le dosage du glucose et de l'urée respectivement par les méthodes colorimétriques à la glucose oxydase et à la diacétyl monoxime (Technicon) mises au point au laboratoire (MICHEL, I971). Les acides aminés libres étaient isolés de 1'extrait selon une technique précédemment décrite (PAWLAK et PION, 1968) et dosés par chromatographie sur résine échangeuse d'ions au moyen d'un analyseur automatique (SPACKMAN, STEIN et MOORE, 1958).

\section{RÉSULTATS}

\section{Consommation d'aliments (fig. I)}

La consommation des granulés d'orge a peu varié durant l'ensemble de l'expérience surtout chez les animaux du groupe B pour lesquels elle a toujours été nettement plus importante que celle des animaux du groupe $\mathrm{A}$. Ces derniers en ont moins consommé en période I qu'en période 5 .

Les quantités de concentrés expérimentaux consommées ont varié en fonction des quantités offertes, qui dépendaient directement de la production laitière, et de l'importance des refus. C'est ce dernier facteur qui a été le plus important en ce qui concerne les différences observées entre périodes; les quantités de concentrés expérimentaux refusées étaient plus faibles au cours des périodes 2 et 4 que durant les périodes $I, 3$ et 5 .

Les animaux du groupe $B$ ont nettement moins consommé de concentrés expérimentaux que ceux du groupe $\mathrm{A}$, particulièrement au cours de la $5^{\mathrm{e}}$ période ; ceci est en partie dû au fait que les quantités offertes étaient moins importantes compte tenu de la production laitière plus faible de ces chèvres. Le concentré contenant 2 p. roo d'urée semble avoir été le mieux accepté par les animaux des deux lots. Les quantités d'urée ingérées figurent sur le tableau 3.

Les mesures des quantités de foin consommées sont assez imprécises compte tenu d'un certain gaspillage difficile à éviter avec les animaux et les installations 
expérimentales utilisées. Il apparaît cependant que les quantités de foin ingérées aient varié dans le même sens que celles des concentrés expérimentaux, mais avec des écarts moindres, sauf pour le lot B pour lequel la consommation est plus faible pendant la $2^{\mathrm{e}}$ période que pendant la première.

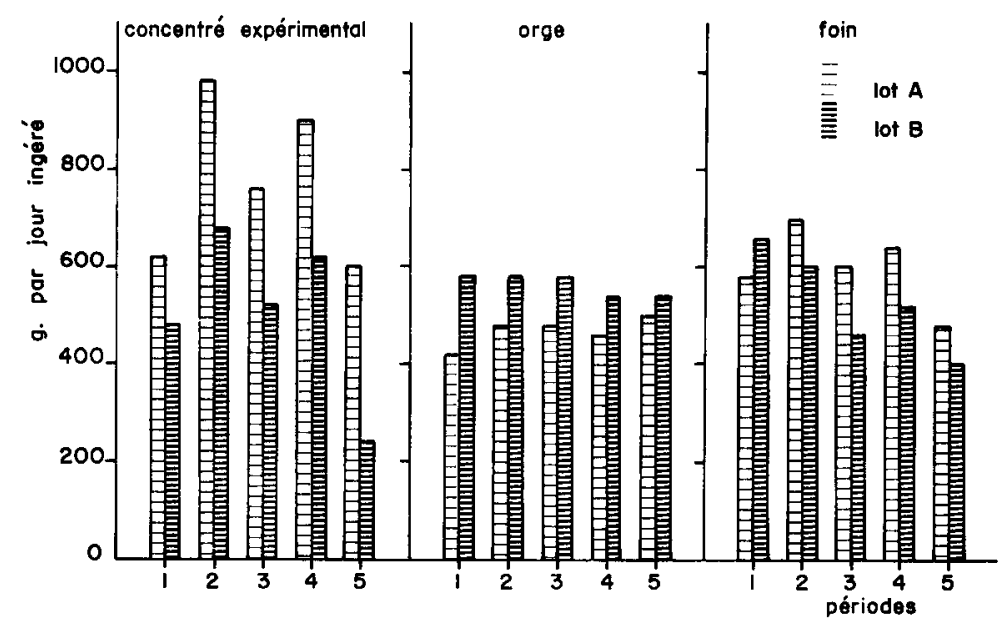

FIG. I. - Consommation d'aliments

TABLEAU 3

Consommation d'urée

\begin{tabular}{c|c|c|c|c|c|c}
\hline \hline Période expérimentale & Lots & 1 & 2 & 3 & 4 & 5 \\
\hline & & & & & & \\
\hline & $\mathrm{A}$ & 6,2 & 19,6 & 22,8 & 0 & 0 \\
Urée ingérée (g/jour) & $\mathrm{B}$ & 4,8 & 13,6 & 15,6 & 0 & 0 \\
\hline Azote uréique & $\mathrm{A}$ & 7,9 & 17,8 & 24,2 & 0 & 0 \\
$\%$ N total ingéré & $\mathrm{B}$ & 6,1 & 15,0 & 20,4 & 0 & 0 \\
\hline \hline
\end{tabular}

Poids des animaux (tabl. 4)

Pendant les 3 premières semaines de l'expérience, les animaux pesant en moyenne $53 \mathrm{~kg}$ pour le lot $\mathrm{A}$ et $48 \mathrm{~kg}$ pour le lot $\mathrm{B}$ ont en moyenne perdu de $2 \mathrm{à} 3 \mathrm{~kg}$, puis ont repris progressivement leurs poids de départ au cours de la période 2 . A la fin de la période $I$, les animaux du groupe $B$ ont vu leur poids augmenter légèrement alors que ceux du groupe $A$ n'ont pratiquement pas varié. Pendant la $2^{\mathbf{e}}$ période, les animaux ont gagné respectivement 5 et $3 \mathrm{~kg}$ pour les groupes $A$ et $B$ alors que leur poids est resté sensiblement constant pendant la période 3 pour les deux lots. Pendant la $4^{\mathrm{e}}$ période, les augmentations de poids ont atteint 3 et $5 \mathrm{~kg}$ respectivement pour les lots $\mathrm{A}$ et $\mathrm{B}$. Le maximum du poids vif des animaux des deux lots a été obtenu au milieu de la $5^{\mathrm{e}}$ période, il a été suivi d'une perte de poids de $\mathrm{I}, 5$ et I $\mathrm{kg}$ respecti- 
TABIEAU 4

Variations du poids vif et productions laitières moyennes des chèvres

\begin{tabular}{|c|c|c|c|c|c|c|}
\hline Période expérimentale & Lots & 1 & 2 & 3 & 4 & 5 \\
\hline $\begin{array}{l}\text { Poids vif des animaux } \\
\qquad(\mathrm{kg})\end{array}$ & $\begin{array}{l}\mathrm{A} \\
\mathrm{B}\end{array}$ & $\begin{array}{l}49,2 \\
47,4\end{array}$ & $\begin{array}{l}54,3 \\
50,4\end{array}$ & $\begin{array}{l}54,0 \\
50,1\end{array}$ & $\begin{array}{l}56,9 \\
55,1\end{array}$ & $\begin{array}{l}55,6 \\
54,0\end{array}$ \\
\hline $\begin{array}{l}\text { Production laitière } \\
\text { (kg/jour) }\end{array}$ & $\begin{array}{l}\text { A } \\
\text { B }\end{array}$ & $\begin{array}{l}2,62 \\
2,15\end{array}$ & $\begin{array}{l}2,58 \\
1,83\end{array}$ & $\begin{array}{l}2,45 \\
1,49\end{array}$ & $\begin{array}{l}2,09 \\
1,28\end{array}$ & $\begin{array}{l}1,66 \\
1,17\end{array}$ \\
\hline $\begin{array}{l}\text { Production laitière }\left(^{2}\right) \\
(\mathrm{kg} / \text { jour })\end{array}$ & $\begin{array}{l}\text { A } \\
\text { B }\end{array}$ & $\begin{array}{l}2,28 \\
1,95\end{array}$ & $\begin{array}{l}2,58 \\
1,71\end{array}$ & $\begin{array}{l}2,24 \\
1,37\end{array}$ & $\begin{array}{l}1,90 \\
1,29\end{array}$ & $\begin{array}{l}1,62 \\
1,05\end{array}$ \\
\hline Taux butyreux $\left({ }^{2}\right)$ & $\begin{array}{l}\text { A } \\
B\end{array}$ & $\begin{array}{l}32,5 \\
30,0\end{array}$ & $\begin{array}{l}27,5 \\
28,5\end{array}$ & $\begin{array}{l}29,5 \\
29,5\end{array}$ & $\begin{array}{l}31,7 \\
33,2\end{array}$ & $\begin{array}{l}33,2 \\
38,0\end{array}$ \\
\hline $\begin{array}{l}\text { Taux de matières azotées } \\
\quad(\mathrm{g} \text { de } \mathrm{N} \times 6,25 / \mathrm{kg} \text { ) }\end{array}$ & $\begin{array}{l}\text { A } \\
\text { B }\end{array}$ & $\begin{array}{l}28,7 \\
28,7\end{array}$ & $\begin{array}{l}26,9 \\
26,6\end{array}$ & $\begin{array}{l}29,0 \\
28,5\end{array}$ & $\begin{array}{l}31,9 \\
31,4\end{array}$ & \\
\hline $\begin{array}{l}\text { Matières azotées } \\
\text { produites dans le lait } \\
\text { (g } \mathrm{N} \times 6,25 / \text { jour })\end{array}$ & $\begin{array}{l}\text { A } \\
\text { B }\end{array}$ & $\begin{array}{l}65,4 \\
56,0\end{array}$ & $\begin{array}{l}69,4 \\
45,5\end{array}$ & $\begin{array}{l}65,0 \\
39,0\end{array}$ & $\begin{array}{l}60,6 \\
40,5\end{array}$ & \\
\hline
\end{tabular}

(1) Détermination sur l'ensemble de la période expérimentale.

(2) Détermination sur la dernière semaine de chaque période (sur l'avant-dernière semaine de la $5^{\mathrm{e}}$ période).

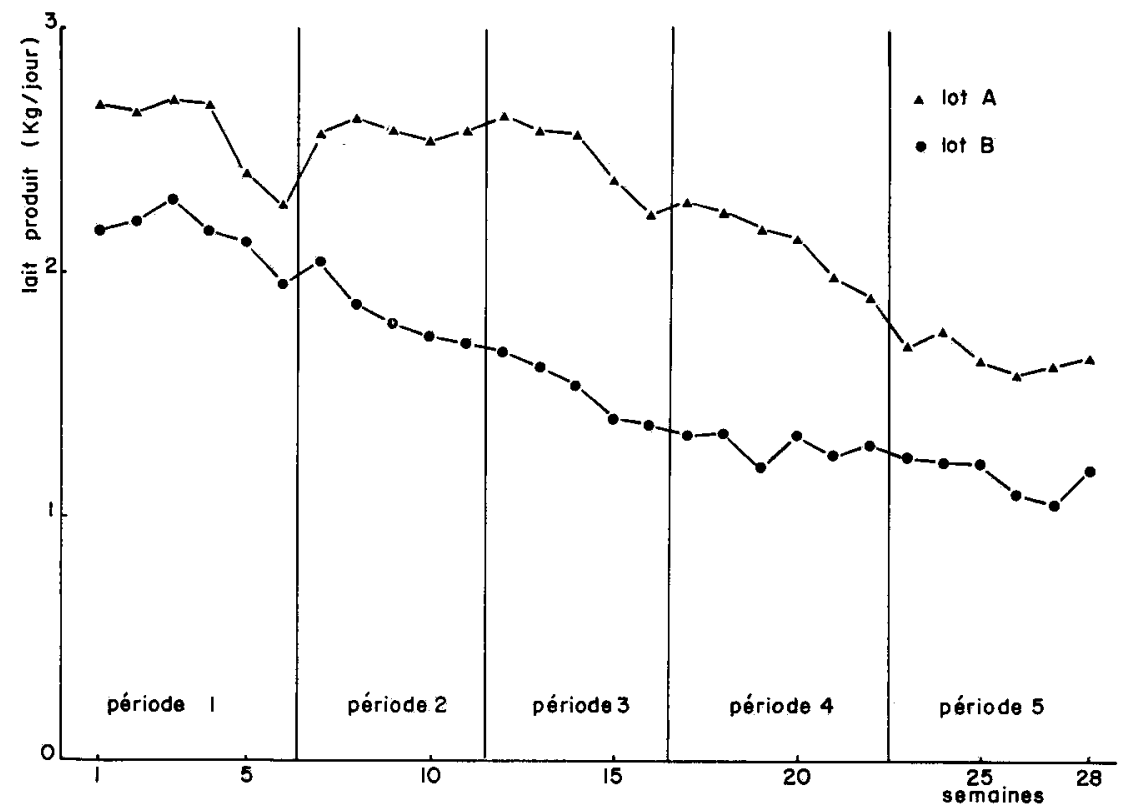

FIG. 2. - Evolution journalière de la production laitière 
vement pour les lots $A$ et $B$. La différence moyenne de poids vif entre le minimum et le maximum a été élevée $(8 \mathrm{~kg})$, ce qui correspond à un gain journalier d'environ $48 \mathrm{~g}$ pendant les 24 premières semaines de l'expérience.

\section{Production laitière}

La courbe de production laitière des animaux du groupe $B$ décroît régulièrement pendant toute la durée de l'expérience alors que celle des chèvres du lot $\mathrm{A}$ ne commence à décroître de manière importante qu'à la fin de la $3^{e}$ période (fig. 2.) Ein fin de première période, la quantité de lait produite par les chèvres du lot $\mathrm{A}$ diminue de manière importante, puis augmente au cours de la deuxième période. Si l'on tient compte de la moyenne journalière des quantités de lait produites sur l'ensemble de chaque période, on constate que la distribution des trois régimes successifs contenant de l'urée n'a pas provoqué de baisse rapide de la production laitière des animaux du lot A (tabl. 4).

L'étude du taux butyreux et du taux de matières azotées mesurés en dernière semaine de chaque période, montre une évolution identique pour chaque lot. Les teneurs sont minimales en fin de $2^{\text {e }}$ période, puis augmentent dans la suite de l'expérience.

\section{Glucose, urée et acides aminés libres du sang}

\section{Glucose et urée sanguins (tabl. 5)}

Les glycémies sont minimales à la fin de la $2^{\mathrm{e}}$ période pour les 2 lots d'animaux, et maximales à la fin de la $4^{\mathrm{e}}$ période pour le lot $\mathrm{B}$ et à la fin de la $5^{\mathrm{e}}$ période pour le lot $A$; la valeur obtenue dans ce dernier cas est particulièrement élevée.

Les teneurs en urée sanguine sont assez élevées mais sont les plus fortes avec les rations ne comprenant pas d'urée, en particulier pour les animaux du lot $B$.

\section{Acides aminés libres}

Les résultats présentés (tabl. 5) ne concernent pas tous les composés détectés sur les chromatogrammes mais seulement les 18 acides aminés qui ont été dosés quantitativement, ainsi que la glutamine et l'asparagine. Ces deux amides ne sont pas séparés, mais le mélange est constitué principalement de glutamine, car la teneur en asparagine du sang est relativement faible. Le tryptophane n'a pas été dosé et la cystine n'a été trouvée qu'à l'état de traces dans les échantillons analysés.

La somme des concentrations des acides aminés est toujours plus faible dans le cas des animaux du lot $\mathrm{A}$ que chez ceux du lot $\mathrm{B}$ sauf pendant la $5^{\mathrm{e}}$ période où les différences entre les deux lots sont très faibles. Pour les deux lots, le maximum est observé en fin de première période, et le minimum en fin de $4^{\mathrm{e}}$ période pour le lot $\mathrm{A}$ et en fin de $5^{\mathrm{e}}$ période pour le lot $\mathrm{B}$.

La somme des concentrations des acides aminés considérés comme indispensables et semi-indispensables chez les monogastriques est plus faible dans le cas des chèvres du lot A que dans celui des animaux du lot B. Elle présente, dans le cas du lot A, un maximum en fin de $4^{\mathrm{e}}$ période et un minimum en fin de $2^{\mathrm{e}}$ période. Pour le lot $B$, les variations au cours des différentes périodes sont moins importantes, mais un minimum peut être observé en fin de $3^{e}$ période. 
La teneur en valine à la fin de la $5^{\mathrm{e}}$ période est plus élevée dans le sang des animaux du groupe $B$ que la moyenne des valeurs obtenues pour cet acide aminé sur l'ensemble de l'expérience. Il en est de même à la fin de la première période pour 1'isoleucine et moins nettement pour la leucine à la fin des $\mathrm{I}^{\mathrm{re}} \mathrm{e}^{\mathrm{t}} 5^{\mathrm{e}}$ périodes. Au contraire, ces trois acides aminés à chaînes ramifiées sont en concentrations relativement faibles dans le sang des animaux du groupe $B$ en fin de $3^{\mathrm{e}}$ période, c'est-à-dire lorsque le concentré contenant la proportion d'urée la plus élevée était distribué.

\section{TABLEAU 5}

Aminoacidémie libre, urémie et glycémie des chèvres (mg/roo $\mathrm{g}$ de sang frais)

\begin{tabular}{|c|c|c|c|c|c|c|c|c|c|c|}
\hline \multirow{2}{*}{$\frac{\text { Lots d'animaux }}{\text { Période expérimentale }}$} & \multicolumn{5}{|c|}{$\mathbf{A}$} & \multicolumn{5}{|c|}{ B } \\
\hline & 1 & 2 & 3 & 4 & 5 & 1 & 2 & 3 & 4 & 5 \\
\hline Acide aspartique . & 0,15 & 0,10 & 0,07 & 0,11 & 0,14 & 0,10 & 0,15 & 0,07 & 0,05 & 0,10 \\
\hline Thréonine ............ & 0,54 & 0,37 & 0,44 & 0,46 & 0,55 & 0,51 & 0,53 & 0,44 & 0,48 & 0,49 \\
\hline Sérine $\ldots \ldots \ldots \ldots \ldots \ldots$ & 1,39 & 1,18 & 0,95 & 0,96 & 1,25 & 1,10 & 1,25 & 1,19 & 1,09 & 0,78 \\
\hline Glutamine + Asparagine . . & 3,84 & 4,29 & 4,03 & 3,28 & 3,00 & 3,86 & 3,87 & 3,90 & 3,38 & 2,38 \\
\hline Acide glutamique $\ldots \ldots \ldots$ & 1,14 & 1,14 & 0,83 & 0,88 & 1,09 & 1,16 & 0,90 & 1,00 & 1,00 & 0,76 \\
\hline Froline $\ldots \ldots \ldots \ldots \ldots$ & 1,41 & 1,22 & 1,41 & 1,25 & 1,37 & 1,42 & 1,50 & $1, \mathbf{4} 1$ & 1,47 & 1,14 \\
\hline Citrulline $\ldots \ldots \ldots \ldots \ldots$ & 1,80 & 2,14 & 1,73 & 1,66 & 1,82 & 1,76 & 1,64 & 1,56 & 1,86 & 2,51 \\
\hline Glycine $\ldots \ldots \ldots \ldots \ldots$ & 6,08 & 5,11 & 4,57 & 4,08 & 4,87 & 5,06 & 4,79 & 4,96 & 4,73 & 4,74 \\
\hline Alanine & 1,58 & 1,21 & 1,23 & 1,09 & 1,41 & 1,52 & 1,32 & 1,39 & 1,34 & 0,95 \\
\hline Valine... & 1,31 & 1,32 & 1,22 & 1,34 & 1,21 & 1,57 & 1,78 & 1,31 & 1,50 & 1,90 \\
\hline Méthionine . & 0,54 & 0,30 & 0,49 & 0,53 & 0,47 & 0,33 & 0,38 & 0,56 & 0,33 & 0,47 \\
\hline Isoleucine & 0,99 & 0,80 & 0,91 & 0,92 & 0,91 & 1,32 & 1,09 & 0,93 & 1,09 & 1,02 \\
\hline Leucine $\ldots .$. & 0,98 & 0,90 & 0,97 & 1,09 & 0,86 & 1,22 & 1,18 & 0,97 & 1,09 & 1,29 \\
\hline Tyrosine.......... & 0,68 & 0,69 & 0,88 & 0,88 & 0,82 & 0,89 & 0,85 & 0,84 & 0,95 & 0,72 \\
\hline Phénylalanine .. & 0,54 & 0,52 & 0,60 & 0,63 & 0,61 & 0,70 & 0,56 & 0,64 & 0,70 & 0,54 \\
\hline Ornithine .. & 0,48 & 0,61 & 0,51 & 0,62 & 0,40 & 0,67 & 0,85 & 0,61 & 0,78 & 0,55 \\
\hline Lysine . . . . & 1,18 & 1,25 & 1,08 & 1,51 & 1,44 & 1,42 & 1,70 & 1,20 & 1,73 & 1,53 \\
\hline Histidine & 0,56 & 0,36 & 0,61 & 0,68 & 0,60 & 0,69 & 0,58 & 0,62 & 0,58 & 0,67 \\
\hline Arginine $\ldots \ldots \ldots \ldots \ldots$ & 1,31 & 1,11 & 1,34 & 1,29 & 1,19 & 1,94 & 1,36 & 1,54 & 1,29 & 1,65 \\
\hline Somme des acides aminés. & 25,5 & 24,6 & 23,9 & 23,3 & 24,0 & 27,3 & 26,3 & 25,2 & 25,5 & 24,2 \\
\hline $\begin{array}{l}\text { Somme des acides aminés in- } \\
\text { dispensables et semi-indis- }\end{array}$ & & & & & & & & & & \\
\hline pensables $\ldots \ldots \ldots \ldots \ldots$ & 8,6 & 7,6 & 8,5 & 9,3 & 8,7 & 10,6 & 10,0 & 9,1 & 9,7 & 10,3 \\
\hline Somme des acides aminés non & & & & & & & & & & \\
\hline $\begin{array}{l}\text { indispensables et amides } \\
\text { Somme des acides aminés du }\end{array}$ & 17,9 & 17,0 & 15,3 & 13,9 & 15,4 & 16,7 & 16,3 & 16,1 & 15,7 & 13,9 \\
\hline cycle de l'urée...$\ldots \ldots$ & 3,59 & 3,86 & 3,58 & 3,57 & 3,41 & 4,38 & 3,86 & 3,71 & 3,93 & 4,71 \\
\hline Urée & 29 & 28 & 42 & 46 & 46 & 25 & 24 & 24 & 48 & 47 \\
\hline Glucose . & 75 & 39 & 51 & 71 & 112 & 49 & 22 & $2_{t}^{\prime}$ & 96 & 87 \\
\hline
\end{tabular}

Les concentrations sanguines en lysine ont considérablement varié au cours de l'expérience, surtout dans le cas des animaux du lot B. C'est ainsi que pour les deux 
lots de chèvres, le minimum était obtenu à la fin de la $3^{\mathrm{e}}$ période et le maximum à la fin de la $4^{\mathrm{e}}$ période. Il faut, de plus, noter une valeur élevée pour les animaux du groupe $\mathrm{B}$ à la fin de la $2^{\mathrm{e}}$ période.

La teneur en histidine est particulièrement faible dans le sang des animaux du groupe $\mathrm{A}$ à la fin de la $2^{\mathrm{e}}$ période. Les teneurs en arginine ont beaucoup varié d'une période à l'autre chez les chèvres du lot $\mathrm{B}$. Deux valeurs relativement élevées sont observées en fin des périodes I et 5 .

Certains acides aminés "indispensables" se trouvent généralement en faibles concentrations dans le sang des animaux, par comparaison avec ce qui avait été constaté dans d'autres expériences. Il s'agit en particulier de la thréonine, de la valine, de la leucine et de l'histidine. Les teneurs des acides aminés " indispensables " sont en général plus faibles dans le sang des animaux du lot A que chez ceux du lot $B$.

La somme des teneurs en acides aminés " non indispensables" et en amides a tendance à être plus élevée au début qu'en fin d'expérience, mais la valeur obtenue dans le cas des animaux du lot $\mathrm{A}$ en fin de $5^{\mathrm{e}}$ période est supérieure à celles correspondant à la fin des $3^{\mathrm{e}}$ et $4^{\mathrm{e}}$ périodes.

Les teneurs en certains acides aminés " non indispensables » du sang des animaux du groupe $B$ en fin de $5^{e}$ période sont particulièrement faibles par comparaison avec les autres valeurs observées au cours de l'expérience; il s'agit de la sérine, de l'alanine, de la proline, de l'acide glutamique et de la glutamine. Par ailleurs, cette dernière est en concentrations nettement plus élevées chez les animaux des deux lots à la fin des trois premières périodes, c'est-à-dire lorsque les rations distribuées comportaient de l'urée, que lorsque les animaux recevaient le concentré comportant la plus forte proportion de tourteau d'arachide.

La quantité de glycine libre dans le sang des chèvres du groupe $A$ décroît rapidement au cours des quatre premières périodes de l'expérience pour augmenter ensuite en fin de la $5^{\text {e }}$ période.

Les teneurs en citrulline sont remarquablement élevées en fin de $2^{\mathrm{e}}$ période pour le lot $\mathrm{A}$ et surtout en fin de $5^{\mathrm{e}}$ période pour le lot $\mathrm{B}$. La somme des teneurs en acides aminés du cycle de l'urée est maximale pour ce même prélèvement, ce qui est dût aux concentrations en arginine et en citrulline.

\section{DISCUSSION}

Les quantités d'aliments distribuées étaient calculées en fonction de la production laitière, avec une marge de sécurité. En fait, le taux de satisfaction des besoins n'a pas été le même pour toutes les périodes expérimentales, par suite de l'importance variable des refus (tabl. 6). La diminution sensible de la production laitière des animaux du lot $\mathrm{A}$ en fin de première période peut s'expliquer par le fait que les besoins azotés et énergétiques de ces chèvres n'étaient pas correctement satisfaits.

Le fait d'avoir utilisé des régimes comportant une proportion élevée de céréale par rapport au foin, ce qui est inhabituel dans le cas de l'alimentation des chèvres, peut expliquer l'importante prise de poids des animaux constatée durant les 24 premières semaines de l'expérience. En effet, il a été observé chez les vaches laitières (RÉmond et JOURNET, I97I et I972) que l'orge tend à orienter les fermentations dans 
le sens d'une production plus importante d'acide propionique dans le rumen, à la différence du foin qui favorise la production d'acide acétique. Or, ce dernier est utilisé par l'animal hôte pour la synthèse d'une partie des matières grasses du lait, alors que l'acide propionique favoriserait davantage le dépôt de lipides corporels.

\section{TABLEAU 6}

Estimation du degré de satisfaction des besoins azotés et énergétiques des chèvres

\begin{tabular}{c|c|c|c|c|c|c}
\hline \hline Période expérimentale & Lots & 1 & 2 & 3 & 4 & 5 \\
& & & & & & \\
\hline & & & & & & \\
M.A.D. : Ingéré/besoins & $\mathrm{A}$ & - & + & + & ++ & + \\
\hline U.F. : Ingéré/besoins & $\mathrm{B}$ & 0 & ++ & ++ & ++ & - \\
\hline
\end{tabular}

La production laitière des animaux du groupe B a constamment diminué au cours des cinq périodes expérimentales, alors que celle des animaux du lot A est restée globalement constante pendant les 3 premières périodes pour diminuer ensuite. Cela ne semble pas s'expliquer par le stade de lactation, puisque au début de l'expérience, ces animaux étaient en production depuis environ 2 mois, et que la quantité de lait produite par les animaux du lot A n'a pas diminué nettement pendant les 4 premiers mois de lactation. Le taux relativement élevé de céréale dans la ration a pu avoir une influence plus marquée chez les animaux du lot $B$, soit parce que leur potentiel de production laitière était le plus faible, soit parce que le changement d'alimentation au début de l'expérience avait été plus marqué chez ces animaux que chez les autres.

Les taux d'urée sanguine relativement peu importants observés lorsque les animaux consomment les concentrés contenant de 1'urée montrent que la libération d'ammoniac dans le rumen n'a pas été trop rapide par rapport à la fourniture d'énergie pour les synthèses bactériennes, ou qu'un mécanisme de régulation a permis une utilisation importante de l'ammoniac provenant du rumen. Le fait que l'ingestion d'urée ait pour conséquence des taux relativement élevés de glutamine sanguine permet de penser que le passage d'ammoniac du rumen dans le sang a effectivement été augmenté, et que les microorganismes en ont probablement utilisé une proportion plus faible que dans le cas où les animaux recevaient uniquement de l'arachide comme complément azoté. La glutamine a pu être formée à partir d'acide glutamique et d'ammoniac dans la paroi du rumen. Par ailleurs, le fait que les taux de glucose sanguin les plus faibles soient généralement observés dans le cas des régimes les plus riches en urée peut être dû à une utilisation plus rapide par la flore des produits d'hydrolyse de l'amidon, liée à la grande vitesse de libération de l'ammoniac à partir de l'urée dans le rumen, ou à une synthèse d'acides aminés non indispensables par l'animal lui-même.

Les dosages d'acides aminés libres ont été effectués à partir d'échantillons pré- 
levés matin et soir le même jour, au cours de la dernière semaine de chaque période expérimentale. Cette méthode a été adoptée à la suite d'une expérience réalisée avec des vaches laitières (CHAMPREDon, Pion et FAUCONNEAU, I969 $a$ ) montrant que l'amplitude journalière des variations de concentration des acides aminés libres dans le sang des ruminants était relativement faible. D’autre part, les échantillons prélevés individuellement ont été regroupés par lots de chèvres et par périodes expérimentales compte tenu du fait que les aminoacidémies libres d'animaux placés dans les mêmes conditions expérimentales sont peu différentes (CHAMPREDON, I972).

La part des acides aminés "indispensables et semi-indispensables " dans l'ensemble des acides aminés est nettement plus faible dans les échantillons prélevés au cours de la présente expérience que dans ceux qui avaient été obtenus chez des animaux à l'entretien ; elle peut, dans ce cas, atteindre et même parfois dépasser 50 p. Ioo de la somme (Champredon, Pion et Fauconneau, Ig69 $b$ ). C'est pendant les périodes où la production était la plus faible que l'on a observé le pourcentage le plus élevé surtout pour le lot $B$; en revanche, pendant les périodes où les animaux du lot $A$ fournissaient les plus grandes quantités de lait les pourcentages ont été les plus faibles. Ceci semble mettre en évidence le rôle prépondérant de la glande mammaire qui capte d'autant plus d'acides aminés "indispensables » dans le sang que la synthèse des protéines du lait est plus importante.

La faible teneur en histidine du sang des animaux du lot $\mathrm{A}$, en fin de $2^{\circ}$ période, pourrait s'expliquer par le fait que cet acide aminé est incorporé en quantité notable dans les protéines du lait, alors qu'il se trouve en proportion relativement faible dans les matières azotées des contenus de duodénum.

Il semble que les concentrations des divers acides aminés libres dans le sang varient plus en fonction du rapport entre la production des animaux et leurs niveaux d'alimentation énergétique et azotée qu'en fonction de la substitution d'urée au tourteau d'arachide : c'est en effet entre la $4^{e}$ et $1 a 5^{e}$ période, alors que les chèvres recevaient le même concentré expérimental mais ingéraient des quantités très différentes d'aliments, que l'on observe les écarts les plus importants dans les teneurs en certains acides aminés libres du sang notamment pour le groupe $B$.

L'influence de la présence d'urée dans le régime semble plus faible que celle qui avait été constatée chez des vaches laitières recevant de l'ensilage de maïs (CHAMPREDON, Pion et Journer, I970) : chez ces animaux, les concentrations de la plupart des acides aminés " indispensables » du sang étaient plus faibles lorsqu'ils ingéraient de l'urée que lorsqu'ils recevaient du tourteau d'arachide en complément de la ration de base ; il faut noter, toutefois, que les écarts observés n'étaient jamais très importants, et que les teneurs étaient sensiblement plus élevées lorsque les animaux ont ultérieurement reçu de 1 'herbe que quand ils consommaient les rations expérimentales.

Les proportions relativement faibles de l'azote uréique dans l'azote total ingéré (tabl. 3) expliquent que nos résultats soient différents de ceux qui ont été obtenus par VirTanein (I966) dans le cas de vaches laitières et par Ortuen et Putnam (I966) dans le cas de bovins en croissance ; ces auteurs ont constaté que l'utilisation d'urée comme seule source d'azote dans les rations provoquait une baisse notable des teneurs en certains acides aminés « indispensables » du plasma.

L'influence du taux de satisfaction des besoins énergétiques et azotés est particulièrement mis en évidence au cours de la $5^{\mathrm{e}}$ période dansle cas des animaux du lot $B$ : les teneurs en certains acides aminés « non indispensables » et en tyrosine sont faibles, 
ce qui peut être dû à l'utilisation de ces composés comme sources d'énergie pour compenser la relative sous-alimentation énergétique des chèvres ; les augmentations des concentrations de l'arginine et de la citrulline sont les témoins de l'activation du cycle de l'urée par suite des désaminations associées à l'utilisation énergétique de chaînes carbonées. La carence azotée associée à la carence énergétique semble entraîner un certain catabolisme protéique qui se manifeste en particulier par une augmentation des teneurs du sang en leucine et en valine ainsi que cela a été constaté chez le porc (CUPERLOVIC et STOSIC, I970), ces acides aminés étant peu catabolisés.

Les résultats obtenus sont en accord avec un certain nombre d'études sur la composition en acides aminés des matières azotées des contenus digestifs qui quittent le rumen pour pénétrer dans l'intestin grêle.

PotTER, LiTTLLE et Mitcheli (I969), ont montré que les proportions molaires des acides aminés dans les hydrolysats de contenus de caillette de bovins étaient très voisines lorsque les animaux recevaient de l'urée ou du tourteau de soja distribués en complément d'une ration à base de maïs. ORSKOV, FrasER et Mc DoNaLD (I97I) ont étudié l'influence, sur la composition en acides aminés des contenus de caillette, d'un apport croissant d'urée ou de farine de poisson dans une ration d'orge distribuée à des brebis ; les teneurs de certains acides aminés augmentent dans le cas de l'apport complémentaire de farine de poisson, mais les résultats obtenus différent généralement peu de ceux correspondant à l'apport d'urée. Enfin, Champredon, Pion et THIVEND (I97I) ont montré que les compositions en acides aminés des matières azotées des contenus de duodénum de bovins étaient relativement voisines lorsque les animaux recevaient des rations très différentes les unes des autres (enlisage de maïs-urée foin-orge-urée, herbe). Tous ces résultats montrent que, si les quantités d'azote total et d'azote non protéique quittant la caillette et le rumen peuvent varier avec le régime (urée), les proportions relatives des divers acides aminés sont comparables ; ce qui expliqueraitles faibles différences liées à la nature des matières azotées du régime trouvées au niveau du sang.

\section{CONCLUSION}

Les écarts entre les besoins des animaux, en particulier pour la production laitière, et les quantités d'aliments ingérés, semblent avoir été, dans cette expérience, le principal facteur de variation des teneurs en acides aminés libres du sang des chèvres, et le remplacement du tourteau d'arachide par de l'urée paraît n'avoir eu qu'une influence plus limitée sur l'aminoacidémie libre. Le type de régime utilisé dans cette expérience (beaucoup d'amidon et peu d'azote soluble) semble particulièrement favorable à l'utilisation de l'urée tant par les bactéries que par l'organisme des ruminants. L'ensemble des résultats, qu'il s'agisse des quantités d'aliments consommés, du poids vif des animaux, de leur production laitière ou des concentrations des différents métabolites sanguins dosés, montre que l'association de $2 \mathrm{p}$. Ioo d'urée et de $4 \mathrm{p}$. Ioo de tourteau d'arachide dans un concentré à base d'orge semble être la plus favorable à la satisfaction des besoins azotés et énergétiques des animaux étudiés. 


\section{REMERCIEMENTS}

Nous remercions M. M.-C. Michel qui a bien voulu effectuer les dosages de glucose et d'urée sanguine.

Ce travail a été réalisé en partie grâce au contrat de la Délégation Générale à la Recherche Scientifique et Technique $\mathrm{n}^{\circ} 6700942$.

\section{SUMMARY}

\section{EFFECT OF UREA CONTENT}

\section{IN THE DIET ON FREE AMINOACIDEMIA IN LACTATING GOATS}

Eight lactating dairy goats received diets of hay, barley, and experimental concentrates during five to six-week periods (table 2). The amount of hay was about one-third of the total diet. The concentrates used contained I, 2, 3, and o p. Ioo, respectively, of trea, and were made isonitrogenous by adding peanut meal (table I). Feed intake, milk production, fat, and protein in milk were recorded. Free amino acids (ion exchange chromatography), urea, and glucose were estimated in blood taken from the jugular vein at the end of each period.

The amount of concentrates ingested varied widely from one period to another (fig. I), the preferred one containing 2 p. I 00 of urea and 4 p. Ioo of peanut meal. The liveweight of animals increased greatly during the experiment (table 4 ) as a result of the high cereal content of the diets. It is assumed that these diets are better for fattening than for milk production. The urea content of the diets had little effect on milk production (fig. 2).

Urea and glucose blood levels were higher when goats ate the concentrates containing no urea (table 5). The amounts of urea nitrogen in the diet (not exceeding $25 \mathrm{p}$. Ioo of total nitrogen) (table 3) seem to be well utilized. Variations of free amino acid concentrations in total blood were smaller than in previous experiments. The differences between food intake and energy and nitrogen requirements of the animals during each period affected free amino acids in the blood more than did urea or peanut meal ratios in the diets. Essential amino acid levels were low in the blood of goats producing the largest amounts of milk, showing a greater uptake of these amino acids by the udder. In a group of goats, large differences in free amino acid patterns were observed between two periods when animals received the same diet but consumed widely different amounts of food (table 6). When they did not eat enough feed to meet their requirements, some non-essential amino acids were used for energetic purposes and were low in the blood. Arginine and citrulline were high as a result of deaminations of other amino acids. The high levels of valine and leucine in the same sample may be explained by tissue protein metabolism.

\section{RÉFÉRENCES BIBLIOGRAPHIQUES}

Armstrong D. G., Trinder N., I966. The use of urea and other non-protein nitrogenous substances in rations for ruminants. J. Univ. Newcastle upon Tyne agric. Soc., 20, $21-33$.

Briggs M. H., I967. Urea as a protein supplement. I st ed. Pergamon Press, Long Island City, New York. Chalupa W., 1968. Problems in feeding urea to ruminants. J. Anim. Sci., 27, 207-219.

Champredon C., Pion R., Fauconneau G., ig69a. L'aminoacidémie libre des bovins. Variations au cours de la journée. C. R. Acad. Sci. Paris Série D, 269, 2029-2032.

Champredon C., Pion R., Fauconneau G., ig69 $b$. L'aminoacidémie libre des chèvies. Variations avec le régime. C. R. Soc. Biol., 163, 2319-2321.

Champredon C., Pion R., Journet M., I97o. Influence de la nature des matières azotées a'imentaires sur l'aminoacidémie libre des vaches laitières. Ann. Biol. anim. Bioch. Biophys., 10, 517-521.

Champredon C., Pron R., Thivend P., r97I. Composition en acides aminés des matières azıtées du contenu duodénal du ruminant. Ann. Biol. anim. Bioch. Biophys., 11, 298-299. 
Champredon C., I972. Thése Doctorat de spécialité (en préparation)

Cuperlovic M., Stosic D., r97o. Some effects of protein deficiency in young growing pigs. II. Blood plasma free amino acids. Acta Vet. Scand., 11, I-I5.

EgAN A. R., Black A. L., I968. Glutamic acid metabolism in the lactating dairy cows. J. Nutr., 96, $450-460$.

Egan A. R., Moller F, Black A, L., I970. Metabolism of glutamic acid, valine and arginine by the lactating goat. J. Nutr., 100,4I9-428.

Fehr P. M., Disset R., 1969. Peut-on alimenter rationnellement les chèvres? Rev. Élevage, $45^{\circ}$ numéro spécial, 145 -I5r.

Helmer L. G., Barteey E. E., I97x. Progress in the utilization of urea as a protein replacer for ruminants. A review. J. Dairy Sci., 54, 25-5x.

Mephan B. T., LiNzell J. L., I966. A. quantitative assessment of the contribution of individual plasma amino acids to the synthesis of milk protein by the goat mammary gland. Biochem. J., 101, $76-83$.

Mrcher M.-C., I97т. Analyse quantitative de quelques substances azotées et glucidiques en milieu biologique. Thèse Doctorat d'Université, Clermont-Ferrand.

OltJen R. R., I969. Effects of feeding ruminants non-protein nitrogen as the only nitrogen source. J. Anim. Sci., 28, 673-682.

Oltjen R. R., Putnam P. A., 1966. Plasma amino acids and nitrogen retention by steers fed purified diets containing urea or isolated soy protein. $J$. Nutr., 89, 385-39I.

orskov E. R., Fraser C., MCDonald I., r97r. Digestion of concentrates in sheep. - 2 The effect of urea or fish meal supplementation of barley diets on the apparent digestion of protein, fat, starch and ash in the rumen, the small intestine and the large intestine, and calculation of volatile fatty acid production. Br. J. Nutr., 25, 243-252.

Pawlak M., PION R., I968. Influence de la supplémentation des proténes du blé par des doses croissantes de lysine sur la teneur en acides aminés libres du sang et du muscle du rat en croissance. $A n n$. Biol. anim, Bioch. Biophys., 8, 5I7-530.

Potter G. D., Little C. O., Mitchell G. E. Jr, ig69. Abomosal nitrogen in steers fed soybean meal or urea.. J. Anim. Sci., 28, 7II-7I3.

RÉmond B., Journet M., I97I. Alimentation des vaches laitières avec des rations à forte proportion d'aliments concentrés. I. Quantités ingérées et production laitière. Ann. Zootech., 20, I69-184.

REMond B., Journet M., I97I. Alimentation des vaches laitières avec des rations à forte proportion d'aliments concentrées. II. Comportement alimentaire et digestion dans le rumen. $A m n$. $Z$ ootech. (sous presse)

Spackman D. H., Stein W. H., Moore J., I958. Automatic recording apparatus for use in the chromatography of amino acids. Analyt. Chem., 30, II90-I206.

Verbeke R., Peeters G., 1965. Uptake of free plasma amino acids by the lactating cow's udder and amino acid composition of the udder lymph. Biochem. J., 94, I83-189.

Virtanen A. I., I966. Milk production of cow's on protein free feed. Science, 153, I603-I6I4. 